\title{
Offloading memory: Serial position effects
}

\author{
Megan O. Kelly ${ }^{1} \cdot$ Evan F. Risko $^{1}$
}

Published online: 3 June 2019

(C) The Psychonomic Society, Inc. 2019

\begin{abstract}
Despite the long history and pervasiveness of cognitive offloading as a memory strategy, the memorial fate of offloaded information is not well understood. Recent work has suggested that offloading information may engage similar mechanisms as instructions to forget (e.g., directed forgetting). In the present investigation, we test this prediction by examining the serial position effect for offloaded information. Previous research has demonstrated that "forget" instructions can eliminate the primacy effect while leaving an intact recency effect. Across two experiments, participants completed a number of free recall trials using an external aid and then a final recall trial without the external aid. We compared a group that was expecting to use the aid for the final trial (offloading) with a group that was not (no-offloading). We found a memory impairment for offloaded items that was characterized by a reduced primacy effect but a typical recency effect, similar to what has been reported in research on intentional/ directed forgetting.
\end{abstract}

Keywords Distributed Memory $\cdot$ Cognitive Offloading $\cdot$ Serial Position Effects $\cdot$ Intentional Forgetting

\section{Introduction}

The limited capacity of our cognitive systems has long led us to offload cognitive demands by integrating our bodies and artifacts in our physical environment into our cognitive acts (e.g., Cherkaoui \& Gilbert, 2017; Dunn \& Risko, 2015; Eskritt \& Ma, 2014; Gilbert, 2015; Gilbert, Bird, Carpenter, Fleming, Sachdeva, \& Tsai 2018; Risko \& Dunn, 2015; Risko, Medimorec, Chisholm, \& Kingstone, 2013; Sparrow, Liu \& Wegner, 2011; Storm \& Stone, 2015). One pervasive form of offloading demands is storing to-be-remembered information externally (e.g., storing important commitments in an agenda; Risko \& Gilbert, 2016). One interesting question that emerges when considering offloading as a memory strategy regards the internal fate of the externally stored information. Recent work demonstrates that offloading to-beremembered information impairs the ability to remember that information in the absence of the external store (e.g., Eskritt \& Ma, 2014; Sparrow et al., 2011). We further examine the fate of offloaded information by investigating the influence of

Megan O. Kelly

mo2kelly@uwaterloo.ca

Evan F. Risko

efrisko@uwaterloo.ca

1 University of Waterloo, Waterloo, Canada serial position in remembering an offloaded list of words unaided.

\section{Offloading impacts memory}

The idea that offloaded information is more readily "forgotten" draws support from recent work by Sparrow et al. (2011) and Eskritt and Ma (2014). Sparrow et al. (2011) tested memory for facts that participants typed into a computer file. Half of the participants were told that the computer would save what they typed (i.e., it would act as an external store), and the other half were told that their information would be erased. Critically, no participant had access to their files at test. Individuals who thought that their typed information was erased had significantly better recall than participants who thought it was saved. Eskritt and Ma (2014) reported similar results. Sparrow et al. (2011) and Eskritt and Ma (2014) likened their findings to forms of intentional/directed forgetting. In intentional/directed forgetting experiments, individuals are presented with items and are told to remember or forget them. When individuals are later tested on all items (including "forget" items), they are less likely to recall forget items than remember items. Multiple explanations for this differential recall have been proposed (e.g., inhibition; Yang, Lei, and Anderson, 2016). One account particularly relevant to the current work is selective rehearsal (Sheard \& MacLeod, 2005). According to this account, items cued as to be remembered are 
rehearsed more than items cued as to be forgotten (Sheard \& MacLeod, 2005). Here, we provide a test of whether or not offloading and intentional/directed forgetting rely on similar mechanisms by examining the dynamics of recall for offloaded information.

\section{Serial position effects}

Free recall tasks consistently produce serial position effects characterized by enhanced recall for beginning-of-list items (primacy) and end-of-list items (recency) relative to middle items (e.g., Glanzer \& Cunitz, 1966; Murdock, 1962). Primacy is typically attributed to differential rehearsal of beginning-of-list items relative to items following (e.g., Fischler, Rundus, \& Atkinson, 1970; Tan \& Ward, 2008). For example, Fischler et al. (1970) showed that participants who freely rehearsed (could differentially rehearse initial list items) were significantly more likely to accurately recall beginning-of-list items (i.e., show primacy) than participants who only rehearsed the current item one at a time (i.e., not differentially). Primacy might also reflect differentially allocated attention to beginning-of-list items compared with later items (Azizian \& Polich, 2007; Sederberg et al., 2006). Recency, however, has often been attributed to end-of-list items being retained in an activated, more accessible state, allowing for enhanced recall (e.g., items remain in shortterm memory; Azizian \& Polich, 2007; Davelaar, GoshenGottstein, Ashkenazi, Haarmann, \& Usher, 2005; Glanzer \& Cunitz, 1966; Shriffin \& Atkinson, 1969). Alternatively, recency may also reflect a greater likelihood of retrieving the more similar temporal context associated with end-of-list items (compared with earlier items) during recall (Sederberg, Howard, \& Kahana, 2008).

Intentional/directed forgetting instructions can affect the form of the serial position function in recall. Lee (2013) presented participants with lists of words and had each word in the list followed by a "remember" or "forget" cue. This was combined with a depth of processing manipulation where participants judged which of two Chinese characters had more strokes (shallow) or whether the word was good or bad (deep; Lee, 2013). We focus on the shallow processing condition as it is putatively more similar to an offloading scenario. At test, participants were told to recall as many words as they could (Lee, 2013). In the shallow condition, "forget" instructions were associated with a decreased primacy effect (no primacy for forget items), with recency intact (Lee, 2013; see also Bjork \& Woodward, 1973). Thus, "forget" instructions appear to have a relatively selective negative impact on primacy. This impact is consistent with "forget" instructions discouraging rehearsal and/or attention. Although "forget" instructions represent an explicit cue indicating that engaging in activities to enhance future recall is unnecessary, the ability to store information externally (i.e., offload memory) could also provide this cue, implicitly. Individuals may "decide" not to employ mnemonic activities (i.e., rehearsal) when storing information externally. If so, then recalling offloaded information (without the aid) should lead to a reduced primacy effect. The intact recency effect in Lee (2013) suggests that items activated most recently remained accessible at recall, despite "forget" instructions. Again, if offloading is similar to being told to forget, then we might expect an intact recency effect when we offload, suggesting that while individuals forego mnemonic activities such as rehearsal, the recently encountered information remains in a relatively active state.

\section{Current investigation}

We manipulated the ability to offload in a free recall task to examine the serial position effect for offloaded information. Participants performed a series of trials on which they were presented with lists of to-be-remembered words and were told to write them down. On the first three trials, participants were instructed that they would have access to their external store (i.e., the paper on which they wrote the words) during the recall phase, which was, indeed, the case. This was essential in order for participants to develop trust/familiarity with the external store. At the beginning of the final trial, half of the participants were notified that they would not be able to refer to the external store during recall, whereas the other half of participants were not. Critically, no participants were able to access their external store on the final trial. Thus, recall on the final trial contrasts memory for the final list when individuals knew that they had to rely on their internal memory (no-offloading) with memory for the final list when they could ostensibly offload those demands to an external store (offloading). Hence, our critical manipulation focuses on the expectations that participants had about their ability to rely on different memory stores (i.e., external vs. internal). To examine the serial position effects, we focused on recall of the first two, middle two, and final two items across the offloading and no-offloading conditions.

\section{Experiments 1a-b}

Both Experiments 1a and $1 \mathrm{~b}$ were preregistered at https://osf. io/qwcxh/ and https://osf.io/2z6gt/, respectively. Experiment 1a used a fixed order of words within each list, and Experiment $1 \mathrm{~b}$ was a replication of Experiment 1a, but randomized word order over serial position. Otherwise, the experiments were identical and are described together.

\section{Method}

Participants In both experiments, data from 64 participants were collected based on an a priori power analysis with a desired power of $.80(\alpha=.05$, two-tailed $)$ to detect a 
Cohen's $d$ of 0.80 for the interaction between offloading condition and the primacy effect (based on pilot work). Participants were undergraduate students in psychology participating for course credit.

Apparatus Participants sat at individual workstations separated by occlusion screens. Each workstation had pens, a computer, a monitor, headphones, and a file folder.

Stimuli We created four 20-item auditory word lists (available at https://osf.io/zjh25/) using the SenticNet 4 word corpus (Cambria, Poria, Bajpai, \& Schuller, 2016). Words were presented in the same position for each list in Experiment 1a, but were randomized across positions in Experiment $1 \mathrm{~b}$. Lists were counterbalanced across trial position (i.e., 1 to 4 ), though, in Experiment 1a, two counterbalances (of the same offloading condition) were repeated.

Procedure Participants sat approximately 50 centimetres in front of their monitors. Participants followed instructions given by the monitor and the researcher for the duration of the experiment (four trials). Each trial had three components: encoding, a 13.5 second period with the external store inaccessible, and recall. A researcher monitored participants to ensure that instructions were followed (e.g., that no participants used the external store on the final list).

Encoding At the beginning of each trial, the participant was presented with an auditory list of to-beremembered words. Each word was presented one at a time and separated by 4,000 milliseconds. Participants were instructed to write down each word as they heard it onto provided paper. Once all words had been presented, the participants placed their written lists into file folders at their stations so that the external store was out of view. Thirteen-and-a-half seconds was provided to participants to enclose their lists into the folders and understand the on-screen instructions for the following recall task.

Recall Participants were instructed to recall the words that they had heard into a text field on the computer. On the first three trials, they were instructed to refer to their external store (open the file folder to consult their list) in order to aid in recalling all of the words. Critically, on the fourth (final) trial, participants recalled without access to their external store (i.e., paper list). Half of the participants were given notice of this at the onset of the fourth trial by on-screen instructions and by the researcher. The other half of the participants were not given this instruction until after the encoding portion of the fourth trial had already finished. Instead, they saw the instructions right before the recall task that stated they were not to open their folder and use their written list (unlike previous trials). Participants were given 150 seconds to complete this final free recall phase and were debriefed and excused when finished.

\section{Results}

Data from one participant from Experiment 1a and three participants from E1b were not analyzed, as they participated after the stopping rule (i.e., 64; participation was grouped in nature) had been reached. One participant in Experiment 1a was replaced because of technical issues. All other participants were included. There were 46 instances in Experiment 1a (76\% during the final trials) and 35 instances in Experiment $1 \mathrm{~b}$ (74\% during the final trials) wherein participants "recalled" a word not on their list. These instances were excluded from analyses and comprised items from previous lists and items never presented. For each relevant analysis, there were no violations of the Levene's test of homogeneity or Mauchly's test of sphericity. Analyses are focused on final trial recall of the initial two $(1,2)$, middle two $(10,11)$, and final two (19, 20) positions across the offloading and no-offloading conditions. Focussing on this item subset facilitated direct comparisons of primacy and recency effects. For both Experiments 1a and $1 \mathrm{~b}$, participants encoded $>99 \%$ of all words on each of Trials 1, 2, 3 and 4. Mean proportions of recall for the six positions of interest for the first three trials (when participants could rely on external stores) are presented in Table $1 .{ }^{1}$ As expected, performance for these trials was near ceiling (Risko \& Dunn, 2015). All confidence intervals reported are bias corrected accelerated bootstrap 95\% confidence intervals using 10,000 replications. Effect sizes reported are Cohens $d$ (lsr package in R; Navarro, 2015) and generalized eta squared $\left(\eta_{\mathrm{G}}^{2}\right.$; ez package in R; Lawrence, 2016).

Experiment 1a A 2 (condition: no-offloading vs. offloading) $\times$ 3 (position: initial vs. middle vs. final) mixed ANOVA revealed no significant main effect of condition, $F(1,62)=$ 3.63, $p=.062, \eta_{\mathrm{G}}^{2}=.02$, and a significant main effect of position, $F(2,124)=3.52, p=.033, \eta_{\mathrm{G}}^{2}=.04$, qualified by an interaction between condition and position, $F(2,124)=$ $6.92, p=.001, \eta_{\mathrm{G}}^{2}=.07$. This interaction was examined further with two $2 \times 2$ ANOVAs. The first $2 \times 2$ ANOVA assessed primacy by comparing initial versus middle position recall proportions across conditions. The second assessed

\footnotetext{
${ }^{1}$ It is unclear why Trial 1 recall proportions were lower in each experiment. One explanation is that participants were less able to follow instructions during Trial 1 relative to trials following. Nevertheless, this highlights the importance of multiple offloading trials for participants to develop trust and familiarity with the external store, similar to how they may in nonlaboratory settings.
} 
Table 1 Mean proportions of recall across trials wherein participants could rely on their external memory stores

\begin{tabular}{lllll}
\hline & & Trial 1 & Trial 2 & Trial 3 \\
\hline E1a & No-offloading & 93.8 & 97.9 & 98.4 \\
& Offloading & 95.3 & 99.0 & 97.9 \\
\multirow{2}{*}{ E1b } & No-offloading & 88.0 & 99.5 & 99.5 \\
& Offloading & 89.1 & 95.8 & 96.9 \\
\hline
\end{tabular}

recency by comparing middle versus final position recall proportions across conditions. The original preregistration of Experiment 1a to follow up on an interaction was ill considered, thus, we follow the preregistration for Experiment $1 \mathrm{~b}$. Hence, these analyses were not preregistered. For primacy, there were significant main effects of condition, $F(1,62)=$ $8.06, p=.006, \eta_{\mathrm{G}}^{2}=.07$, and position, $F(1,62)=6.63, p=$ $.012, \eta_{\mathrm{G}}{ }^{2}=.05$, qualified by a significant interaction between condition and position, $F(1,62)=5.42, p=.023, \eta_{\mathrm{G}}{ }^{2}=.04$. Paired $t$ tests revealed a significant primacy effect in the nooffloading condition, $t(31)=3.51, p=.001, d=0.62$, but not in the offloading condition, $t(31)=0.17, p=.865, d=0.03$. For recency, there were no significant main effects of condition, $F(1,62)=0.46, p=.499, \eta_{\mathrm{G}}^{2}<.01$, or position, $F(1,62)$ $=3.92, p=.052, \eta_{\mathrm{G}}^{2}=.03$, and no interaction between condition and position, $F(1,62)=2.11, p=.152, \eta_{\mathrm{G}}{ }^{2}=.02$. A parallel set of mixed-effects logistic-regression analyses revealed qualitatively similar results.

Experiment 1b A 2 (condition: no-offloading vs. offloading) $\times$ 3 (position: initial vs. middle vs. final) mixed ANOVA revealed no significant main effect of condition, $F(1,62)=$ $3.67, p=.060, \eta_{\mathrm{G}}^{2}=.02$, and a significant main effect of position, $F(2,124)=16.71, p<.001, \eta_{\mathrm{G}}^{2}=.15$, qualified by a significant interaction between condition and position, $F(2,124)=9.59, p<.001, \eta_{\mathrm{G}}{ }^{2}=.09$. This interaction was examined with two $2 \times 2$ ANOVAs (preregistered). For primacy, there were significant main effects of condition, $F(1,62)=10.42, p=.002, \eta_{\mathrm{G}}^{2}=.09$, and position, $F(1,62)$ $=15.38, p<.001, \eta_{\mathrm{G}}^{2}=.09$, which were qualified by a significant interaction between condition and position, $F(1,62)=$ $8.07, p=.006, \eta_{\mathrm{G}}^{2}=.05$. Paired $t$ tests found a significant primacy effect in the no-offloading condition, $t(31)=4.88, p<$ $.001, d=0.86$, but not in the offloading condition, $t(31)=$ $0.751, p=.459, d=0.13$. For recency, there was no significant main effect of condition, $F(1,62)=0.65, p=.422, \eta_{\mathrm{G}}^{2}<.01$, but a main effect of position was significant, $F(1,62)=31.12$, $p<.001, \eta_{\mathrm{G}}{ }^{2}=.22$, such that the recall proportion of the final items was significantly higher than middle items (final: 0.67 vs. middle: 0.31 ). There was no significant interaction between condition and position, $F(1,62)=2.88, p=.095, \eta_{\mathrm{G}}^{2}$ $=.03$. A parallel set of mixed-effects logistic-regression analyses revealed qualitatively similar results Figure 1 presents the mean proportions of recall by serial position and offloading condition for Experiments 1 and 2.

\section{Exploratory analyses}

Overall effect of offloading Our analyses focused on a subset of item positions; however, when considered across all positions, memory for offloaded items was significantly worse in Experiment 1a, $t(61.61)=3.68, p<.001, d=0.92$ (nooffloading: 0.49 vs. offloading: 0.36 ), and Experiment $1 \mathrm{~b}$, $t(60.97)=4.79, p<.001, d=1.20$ (no-offloading: 0.51 vs. offloading: 0.35 ). Mean proportions of recall across all positions for both experiments are presented in Fig. 2.

Offloading on recency In both experiments, there was a trend suggesting that recency was larger in the offloading condition. This was unanticipated, but was deemed worth examining closer (the following analyses were not preregistered). When combining across experiments, a 2 (condition: no-offloading vs. offloading) $\times 2$ (position: middle vs. final) mixed ANOVA

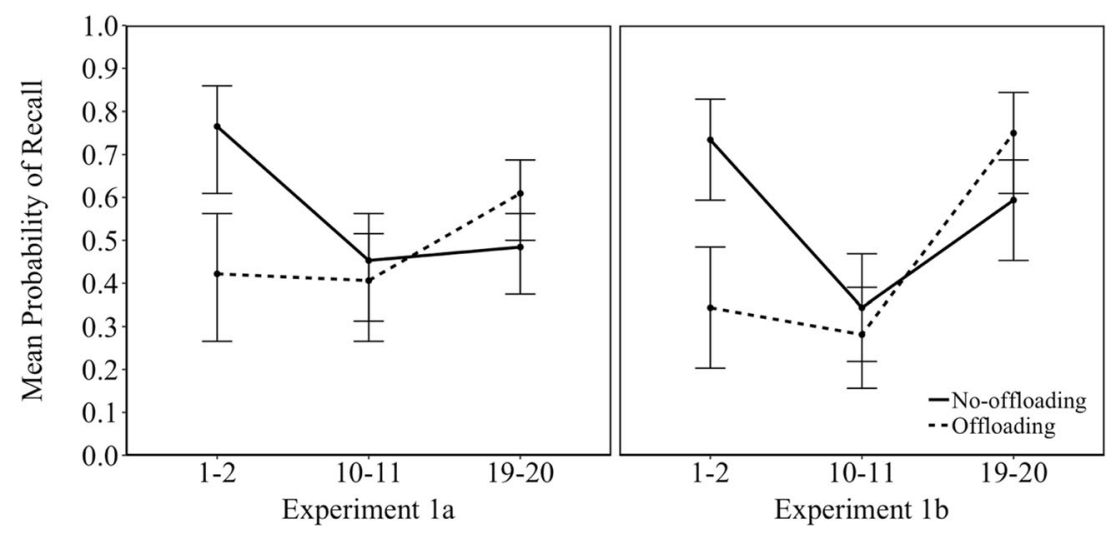

Serial Position

Fig. 1 Mean proportions of recall by serial position, offloading condition, and experiment. Error bars are bias corrected accelerated bootstrap 95\% confidence intervals using 10,000 replications 


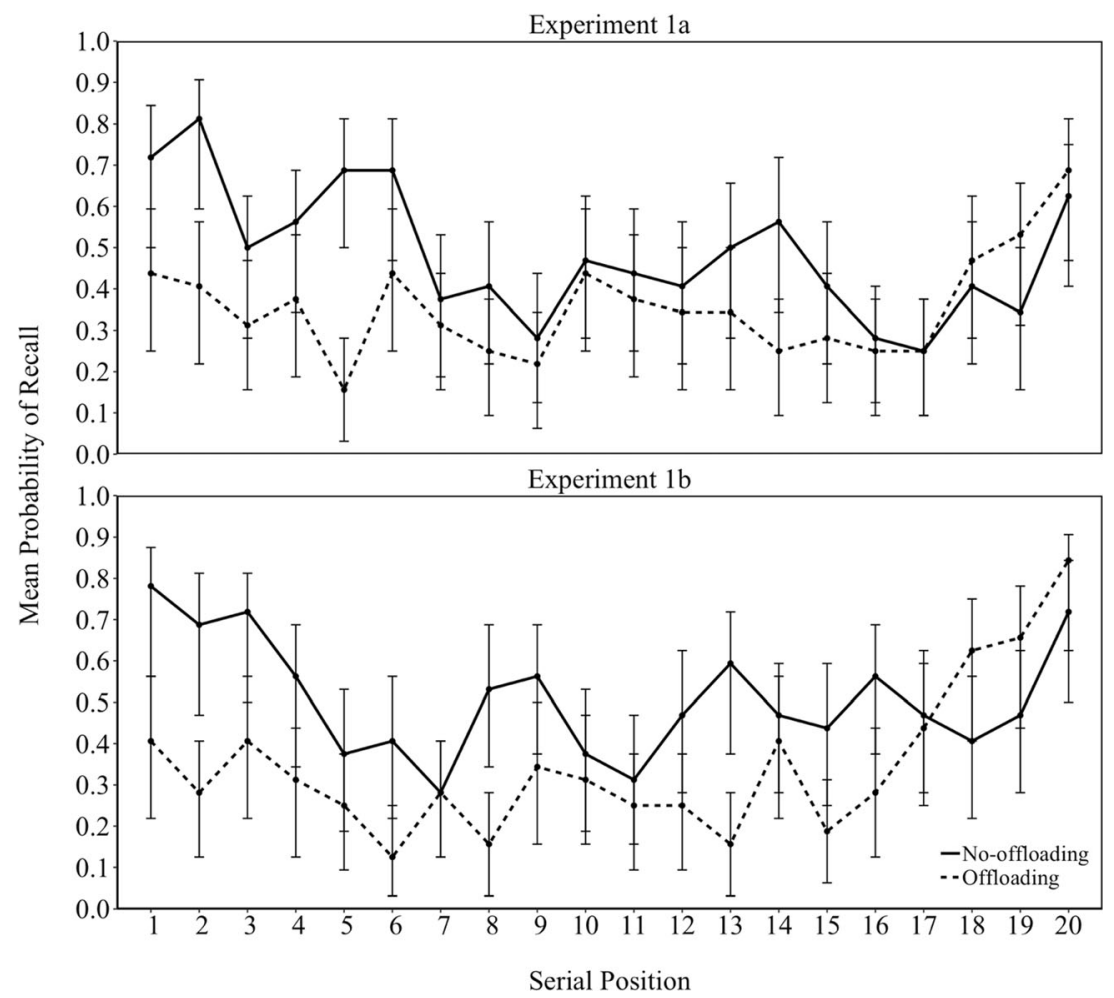

Fig. 2 Mean proportions of recall by item position, offloading condition, and experiment. Error bars are bias corrected accelerated bootstrap 95\% confidence intervals using 10,000 replications

revealed a significant interaction between offloading condition and position, $F(1,124)=4.65, p=.033, \eta_{\mathrm{G}}^{2}=.02$. Welch's two-sample $t$ tests compared offloading with nooffloading recall proportions for middle and final items separately. The recall proportion of the final items was significantly higher in the offloading group than in the no-offloading group (offloading: 0.68 vs. no-offloading: 0.54$), t(125.83)=$ $2.49, p=.014, d=0.44$. The recall proportion of the two middle items was not significantly different between offloading groups (offloading: 0.34 vs. no-offloading: 0.40), $t(125.80)=0.85, p=.395, d=0.15$.

Offloading on primacy versus recency Although the main analyses show that offloading had significant effects on primacy and not recency, a direct statistical comparison is needed to draw the conclusion that offloading influences the primacy effect more than the recency effect (Nieuwenhuis, Forstmann, \& Wagenmakers, 2011; the following analyses not preregistered). We conducted an additional 2 (condition: nooffloading vs. offloading) $\times 2$ (position: initial vs. final) mixed ANOVA for each experiment. When doing so for Experiment 1a, we found a significant main effect of offloading condition, $F(1,62)=4.76, p=.033, \eta_{\mathrm{G}}{ }^{2}=.03$, and no significant main effect of position, $F(1,62)=0.48, p=.493, \eta_{\mathrm{G}}^{2}<.01$, but a significant interaction between offloading condition and position, $F(1,62)=11.88, p=.001, \eta_{\mathrm{G}}^{2}=.11$. Further investigation of the interaction found that in the first position, participants in the no-offloading condition had significantly higher recall than participants in the offloading condition (no-offloading: 0.77 ; offloading: 0.42 ), $t(58.94)=3.60, p=$ $.001, d=0.90$. For the final position, participants in the nooffloading condition had lower recall than participants in the offloading condition (no-offloading: 0.48; offloading: 0.61), though not significantly, $t(61.67)=1.74, p=.087, d=0.44$. A parallel set of mixed-effects logistic-regression analyses revealed qualitatively similar results.

We also conducted the additional 2 (condition: nooffloading vs. offloading) $\times 2$ (position: initial vs. final) mixed ANOVA for Experiment $1 \mathrm{~b}$. We found no significant main effect of offloading condition, $F(1,62)=3.75, p=.057, \eta_{\mathrm{G}}^{2}$ $=.03$, and a significant effect of position, $F(1,62)=4.04, p=$ $.049, \eta_{\mathrm{G}}{ }^{2}=.03$, qualified by a significant interaction between offloading condition and position, $F(1,62)=17.11, p<.001$, $\eta_{\mathrm{G}}{ }^{2}=.13$. Further investigation of the interaction found that in the first position, participants in the no-offloading condition had significantly higher recall than participants in the offloading condition (no-offloading: 0.73 ; offloading: 0.34 ), $t(59.66)=4.17, p<.001, d=1.04$. For the final position, participants in the no-offloading condition had lower recall than participants in the offloading condition (no-offloading: 
0.59; offloading: 0.75$)$, though not significantly, $t(61.94)=$ $1.83, p=.072, d=0.46$. A parallel set of mixed effects logistic regression analyses revealed qualitatively similar results.

\section{Discussion}

We examined serial position effects (e.g., Glanzer \& Cunitz, 1966; Murdock, 1962) for offloaded information as a test of whether offloading mirrors the recall patterns of intentional/ directed forgetting. In both experiments, offloading led to significantly reduced primacy during free recall. Interestingly, offloading did not have any negative impact on recency and appeared (via exploratory analyses combining across experiments) to lead to greater recall of the final items.

Results are consistent with offloading influencing memory in a similar manner to that of "forget" instructions in intentional/ directed forgetting. Critically, both offloading and being instructed to forget lead to a decreased primacy effect. If we take primacy to reflect differential rehearsal (e.g., Fischler et al., 1970; Sederberg et al., 2006; Tan \& Ward, 2008) and/or attention (Azizian \& Polich, 2007; Sederberg et al., 2006), it suggests that offloading discourages one or both of these processes. For example, individuals might encode information to the extent that they record it properly into their external store, but exert no mnemonic effort to remember that information thereafter.

Consistent with Lee (2013), recency was intact when offloading was available. The magnitude of this effect was at least equivalent to that when offloading was unavailable. This suggests (depending on the account of recency) that the encoding of to-be-offloaded information is in an active enough state in memory to produce a robust recency effect (Azizian \& Polich, 2007; Davelaar et al., 2005; Glanzer \& Cunitz, 1966; Shriffin \& Atkinson, 1969) and/or that offloading does not impair the encoding of the temporal context associated with the end-of-list list items (Sederberg et al., 2008). The unexpected finding that memory for the final items was greater in the offloading condition might reflect reduced within-list interference during the final trial when offloading compared with not offloading. If rehearsing beginning-of-list items hinders the encoding of end-of-list items and individuals who offload forego rehearsal, then we might expect greater memory for end-oflist items during offloading (see Storm \& Stone, 2015).

The present investigation focused on the memorial consequences of offloading as an available strategy. Requiring individuals to write down all words (rather than allowing a choice of what to record) seemingly removed the need to "choose" which items to offload. Removing this choice might introduce "unnaturalness" when considering that we typically decide what to offload. However, this seems a necessary compromise when considering those choices as unlikely to be random (see Siegler \& Lemaire, 1997, for discussion). For example, Castel (2008) demonstrated that individuals have some metacognitive awareness of the influence of serial position on memory. If individuals' metacognitions drive their offloading decisions (Dunn \& Risko, 2015; Risko \& Gilbert, 2016), then we might expect serial position to influence offloading choices as well. However, this effect is likely to be small, considering that individuals tend to rely heavily on offloading, even if relying on internal memory would yield comparable performance (Risko \& Dunn, 2015). This raises an interesting question for future research, both in terms of examining the effect of serial position on the choice to offload, and in terms of how providing choice influences one's internal representation of the offloaded (or not) information. Though our chosen form of offloading (writing) represents a common strategy, the emergence of massive digital forms of storage are quickly supplanting it. Previous research has used a mix of external storage types (e.g., digital files, paper and pencil; Eskritt \& Ma, 2014; Hamilton, McIntyre, \& Hertel, 2016; Hertel, 1988; Storm \& Stone, 2015; Risko \& Dunn, 2015; Sparrow et al., 2011). However, we are not aware of any direct comparisons across external store types, thus opening a door to another potentially fruitful line of research.

The present results support the suggestion that offloading memory may engage (or disengage) similar mechanisms as intentional/directed forgetting (Eskritt \& Ma, 2014; Sparrow et al., 2011). Our results are also consistent with reduced topdown rehearsal during offloading, considering the link between primacy and rehearsal (Fischler et al., 1970; Tan \& Ward, 2008). Although we think decreased rehearsal fits with the current data, drawing parallels between intentional/ directed forgetting and offloading raises the interesting question of whether offloading might also have a more active, inhibitory component as some have suggested for intentional/directed forgetting (e.g., Yang et al., 2016). However, when we offload information to an external store, it is likely guided by intentions to have that information for future use. Ostensibly, this is not the case when presented with "forget" instructions - thus, inhibiting offloaded information might be unnecessary.

\section{Conclusion}

The present work revealed that offloading information selectively impaired memory for initial list items and not for later list items. These results are consistent with previous work demonstrating modulation of the serial position curve under "forget" instructions (Lee, 2013) and the hypothesis that offloading and intentional/directed forgetting rely on similar mechanisms.

Acknowledgements This work was supported by a Discovery Grant from the Natural Sciences and Engineering Research Council of 
Canada (NSERC), an Early Researcher Award from the Province of Ontario and funding from the Canada Research Chairs program to E.F.R.

\section{References}

Azizian, A., \& Polich, J. (2007). Evidence for attentional gradient in the serial position memory curve from event-related potentials. Journal of Cognitive Neuroscience, 19(12), 2071-2081.

Bjork, R. A., \& Woodward, A. E. (1973). Directed forgetting of individual words in free recall. Journal of Experimental Psychology, 99(1), 22-27.

Cambria, E., Poria, S., Bajpai, R., \& Schuller, B. (2016). SenticNet 4: A semantic resource for sentiment analyses based on conceptual primitives. Proceedings of COLING 2016, the 26th International Conference on Computational Linguistics: Technical Papers (pp. 2666-2677). Retrieved from https://aclweb.org/anthology/papers/ C/C16/C16-1251/

Castel, A. (2008). Metacognition and learning about primacy and recency effects in free recall: the utilization of intrinsic and extrinsic cues when making judgments of learning. Memory \& Cognition, 36(2), 429-437.

Cherkaoui, M., \& Gilbert, S. J. (2017). Strategic use of reminders in an 'intention offloading' task: Do individuals with autism spectrum conditions compensate for memory difficulties. Neuropsychologia, 97, 140-151.

Davelaar, E. J., Goshen-Gottstein, Y., Ashkenazi, A., Haarmann, H., \& Usher, M. (2005). The demise of short-term memory revisited: Empirical and computational investigations of recency effects. Psychological Review, 117(1), 3-42.

Dunn, T. L., \& Risko, E. F. (2015). Toward a metacognitive account of cognitive offloading. Cognitive Science, 40(5), 1080-1127.

Eskritt, M., \& Ma, S. (2014). Intentional-forgetting: Note-taking as a naturalistic example. Memory \& Cognition, 42, 237-246.

Fischler, I., Rundus, D., \& Atkinson, R. C. (1970). Effects of overt rehearsal procedures on free recall. Psychonomic Science, 19(4), 249250 .

Gilbert, S. J. (2015). Strategic offloading of delayed intentions into the external environment. The Quarterly Journal of Experimental Psychology, 68, 971-992.

Gilbert, S. J., Bird, A., Carpenter, J. M., Fleming, S. M., Sachdeva, C., \& Tsai, P. (2018). Optimal use of reminders: Metacognition, effort, and cognitive offloading. doi:10.31234/osf.io/7fxrg

Glanzer, M., \& Cunitz, A. R. (1966). Two storage mechanisms in free recall. Journal of Verbal Learning and Verbal Behavior, 5, 351-360.

Hamilton, K. A., McIntyre, K. P., \& Hertel, P. T. (2016). Judging knowledge in the digital age: The role of external-memory organization. Applied Cognitive Psychology, 30(6), 1080-1087.

Hertel, P. T. (1988). Monitoring external memory. In M. M. Gruneberg, P. E. Morris, \& R. N. Sykes (Eds.), Practical aspects of memory: Current research and issues (pp. 221-226). New York: Wiley.

Lawrence, M. A. (2016). ez: Easy analysis and visualization of factorial experiments (R Package Version 4.4-0) [Computer software]. Retrieved from https://rdrr.io/cran/ez/

Lee, Y. (2013). Costs and benefits in item-method directed forgetting: Differential effects of encoding and retrieval. The Journal of General Psychology, 140(3), 159-173.
Murdock, B. B. (1962). The serial position effect of free recall. Journal of Experimental Psychology, 64, 482-488.

Navarro, D. J. (2015) Learning statistics with R: A tutorial for psychology students and other beginners (Version 0.5) Adelaide, Australia: University of Adelaide.

Nieuwenhuis, S., Forstmann, B. U., \& Wagenmakers, E. (2011). Erroneous analyses of interactions in neuroscience: A problem of significance. Nature Neuroscience, 14, 1105-1107.

Risko, E. F., \& Dunn, T. L. (2015). Storing information in-the-world: Metacognition and cognitive offloading in a short-term memory task. Consciousness and Cognition, 36, 61-74.

Risko, E. F., \& Gilbert, S. J. (2016). Cognitive offloading. Trends in Cognitive Science, 20(9), 676-688.

Risko, E. F., Medimorec, S., Chisholm, J., \& Kingstone, A. (2013). Rotating with rotated text: A natural behavior approach to investigating cognitive offloading. Cognitive Science, 537-564.

Sederberg, P. B., Gauthier, L. V., Terushkin, V., Miller, J. F., Barnathan, J. A., \& Kahana, M. J. (2006). Oscillatory correlates of the primacy effect in episodic memory. NeuroImage, 32, 1422-1431.

Sederberg, P. B., Howard, M. W., \& Kahana, M. J. (2008). A contextbased theory of recency and contiguity in free recall. Psychological Review, 115(4), 893-912.

Sheard, E. D. \& MacLeod, C. M. (2005). List method directed forgetting: Return of the selective rehearsal account. In N. Ohta, C. Macleod, \& B. Uttl (Eds.), Dynamic cognitive processes (pp. 219-248). Tokyo: Springer-Verlag.

Shriffin, R. M., \& Atkinson, R. C. (1969). Storage and retrieval processes in long-term memory. Psychological Review, 76(2), 179-193.

Siegler, R. S., \& Lemaire, P. (1997). Older and younger adults' strategy choices in multiplication: Testing predictions of ASCM using the choice/no-choice method. Journal of Experimental Psychology: General, 126(1), 71-92.

Sparrow, B., Liu, J., \& Wegner D. M. (2011). Google effects on memory: Cognitive consequences of having information at our fingertips, Science, 333(6043), 776-778.

Storm, B. C., \& Stone, S. M. (2015). Saving-enhanced memory: The benefits of saving on the learning and remembering of new information. Psychological Science, 26(2), 182-188.

Tan, L., \& Ward, G. (2008). Rehearsal in immediate serial recall. Psychonomic Bulletin \& Review, 15(3), 535-542.

Yang, T., Lei, X., \& Anderson, M. A. (2016). Decreased inhibitory control of negative information in directed forgetting. International Journal of Psychophysiology, 100, 44-51.

\section{Open practices statement}

Experiment $1 \mathrm{a}$ and $1 \mathrm{~b}$ were preregistered at $\mathrm{https} / / / \mathrm{osf}$.io/qwexh/ and https://osf.io/2z6gt/, respectively. All data and analysis materials are available at https://osf.io/zjh25/.

Publisher's note Springer Nature remains neutral with regard to jurisdictional claims in published maps and institutional affiliations. 